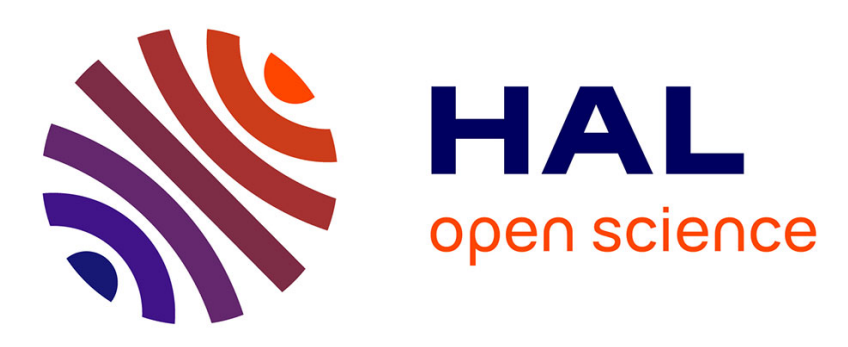

\title{
Kirigami Auxetic Pyramidal Core: Mechanical Properties and Wave Propagation Analysis in Damped Lattice
}

Fabrizio Scarpa, Morvan Ouisse, Manuel Collet, K. Saito

\section{- To cite this version:}

Fabrizio Scarpa, Morvan Ouisse, Manuel Collet, K. Saito. Kirigami Auxetic Pyramidal Core: Mechanical Properties and Wave Propagation Analysis in Damped Lattice. Journal of Vibration and Acoustics, 2013, 135, pp.041001-1 - 041001-11. 10.1115/1.4024433 . hal-00993364

\section{HAL Id: hal-00993364 https://hal.science/hal-00993364}

Submitted on 20 May 2014

HAL is a multi-disciplinary open access archive for the deposit and dissemination of scientific research documents, whether they are published or not. The documents may come from teaching and research institutions in France or abroad, or from public or private research centers.
L'archive ouverte pluridisciplinaire HAL, est destinée au dépôt et à la diffusion de documents scientifiques de niveau recherche, publiés ou non, émanant des établissements d'enseignement et de recherche français ou étrangers, des laboratoires publics ou privés. 


\title{
Kirigami auxetic pyramidal core: mechanical properties and wave propagation analysis in damped lattice
}

\author{
Fabrizio Scarpa* \\ Advanced Composites Centre for Innovation and Science (ACCIS), \\ University of Bristol, BS8 1TR Bristol, UK \\ Morvan Ouisse, Manuel Collet ${ }^{\dagger}$ \\ Institut FEMTO-ST, Département Mécanique Appliquée, \\ UMR CNRS 6174, 25000 Besançon, France \\ Kazuya Saito \\ Institute of Industrial Science (IIS), University of Tokyo, Japan
}

\begin{abstract}
The work describes the manufacturing, mechanical properties and wave propagation characteristics of a pyramidal lattice made exhibiting an auxetic (negative Poisson's ratio) behaviour. Contrary to similar lattice tessellations produced using metal cores, the pyramidal lattice described in this work is manufactured using a Kirigami (Origami plus cutting pattern) technique, which can be applied to a large variety of thermoset and thermoplastic composites. Due to the particular geometry created through this manufacturing technique, the Kirigami pyramidal lattice show an inversion between in-plane and out-of-plane mechanical properties compared to classical honeycomb configurations. Long wavelength approximations are used to calculate the slowness curves, showing unusual zero-curvature phononic properties in the transverse plane. A novel 2D wave propagation technique based on Bloch waves for damped structures is also applied to evaluate the dispersion behaviour of composite (Kevlar/epoxy) lattices with intrinsic hysteretic loss. The 2D wave propagation analysis shows evanescence directivity at different frequency bandwidths and complex modal behaviour due to unusual deformation mechanism of the lattice.
\end{abstract}

\footnotetext{
*Electronic address: f.scarpa@bristol.ac.uk $\dagger$ Electronic address: morvan.ouisse@univ-fcomte.fr

${ }_{\ddagger}^{\ddagger}$ Electronic address: saito-k@iis.u-tokyo.ac.jp
} 


\section{INTRODUCTION}

Lattice pyramidal cores have been developed extensively during the last 10 years, by virtue of novel metal casting techniques that allow producing at relatively low lost large integrated sandwich panels [1,2]. One of the major features of these lattice cores is the high specific strength to failure and in-plane stiffness, which can be used to develop novel energy absorbing integrated cellular structures for static and dynamic loading, and thermal management $[3,4]$.

The pyramidal lattice configuration can be tessellated in a "egg-rack" layout (Figure 1). This particular tessellation is auxetic in the plane. Auxetics indicates a large class of solids and structures exhibiting negative Poisson's ratio - the solid expands rather than contracts when pulled along one direction. Auxetics [5] have been extensively studied for their multifunctional performance, in particular for their tunability in band-gap behaviour [6-8] and aeroelastic tailoring in morphing structures $[9,10]$. The egg-rack tessellation has been identified by Grima et al. as a potential deformation mechanism providing auxeticity in polymer networks built from calix[4]arene blocks [11]. The same tessellation has been identified as existing in bucky-papers with single and multi-walled carbon nanotubes, exhibiting a sign inversion from positive to negative Poisson's ratio when subjected to mechanical loading $[12,13]$.

Three-dimensional complex cellular solids can be also produced using other materials (in particular thermoset and thermoplastic composites) by means of Kirigami techniques. Kirigami is an ancient Japanese art of combining ply-folding techniques (Origami) with cutting patterns to obtain general tessellated and complex structures. Kirigami has been used successfully to produce 3D honeycombs with general shapes using carbon and Kevlar woven fabrics [14], auxetic re-entrant structures [15], and novel morphing wingbox configurations [16]. Figure 2 shows how a Kirigami version of the pyramidal lattice van be fabricated using standard Kevlar 49/914 epoxy preprepgs (Hexcel Composites Ltd, UK). The sheets with the fold line diagram pattern are cut from a single ply of the prepreg by an automated ply cutter (Figure 2a). The sheets with a polyethylene release film are folded in core shapes by using 16 square rods $(20 \times 20 \times 30$ [mm], aluminum - see Figure 2b). The 8 rods are put on the blue cross points of Figure $2 \mathrm{~b}$ from the upper side, and other 8 rods are placed on red ones from the side below (Figure 2c). After placing together these rods like in Figure 
$2 \mathrm{~d}$, the sheet is fixed in the shape of the auxetic pyramidal lattice. The assembly is held by tapes and inserted into a vacuum bag (Figures 2d). The bagged arrangement is put in an autoclave for 60 minutes at $175 \mathrm{C}^{\mathrm{o}}$ to cure the prepreg. The final cured sample is shown in Figure 2e.

The work described in this paper is concerned with the evaluation of the long wavelength properties (first order homogenisation) and detailed two-dimensional wave propagation of the Kirigami pyramidal lattice. The long wavelength approximation is used to derive the effective stress-strain tensor of the homogenised lattice, and to calculate the slowness curves from the eigenvalue problem associated to the Christoffel's equation in various planes. The rationale behind the study of the slowness curves is the identification of possible anomalous behaviour of their curvature. The curvature of the slowness surface has been studied has a metrics to identify caustics in phonon imaging, where the caustics are measured as parabolic lines on the slowness surface [17]. Zero-slowness curves are therefore a symptom of possible solitons created while the wave vector is oriented along angles corresponding to these instability points. To the best of the Authors' knowledge, an extensive study of the homogenised mechanical properties, first order homogenisation and solutions of the Christoffel equation of the particular pyramidal tessellation shown in Figure 2 has not been performed yet. We will show that the lattice is auxetic in the plane, but also possessing a peculiar mechanical behaviour, where the in-plane stiffness is higher than the transverse one - contrary to classical centresymmetric honeycomb configurations existing in open literature. The Kirigami lattice does show indeed zero curvature slowness curves in the transverse plane, depending on the geometric parameters defining the lattice. Moreover, we consider also the case (as in the real engineering application), where hysteretic damping is present in the system, due to the intrinsic loss factor of the prepreg used to manufacture these lattices. A novel wave propagation technique is presented in this work to consider the effect of the damping in the lattice, and identify the directivity of evanescence modes present at different frequencies. While the long wavelength approximation does not reveal any unusual wave propagation pattern in the plane of the lattice, the 2D damped wave propagation techniques highlight the complex modal behaviour existing within the elements of the 3D unit cell structure, and the evanescence directivity due to specific modes dissipating more energy than the others. 


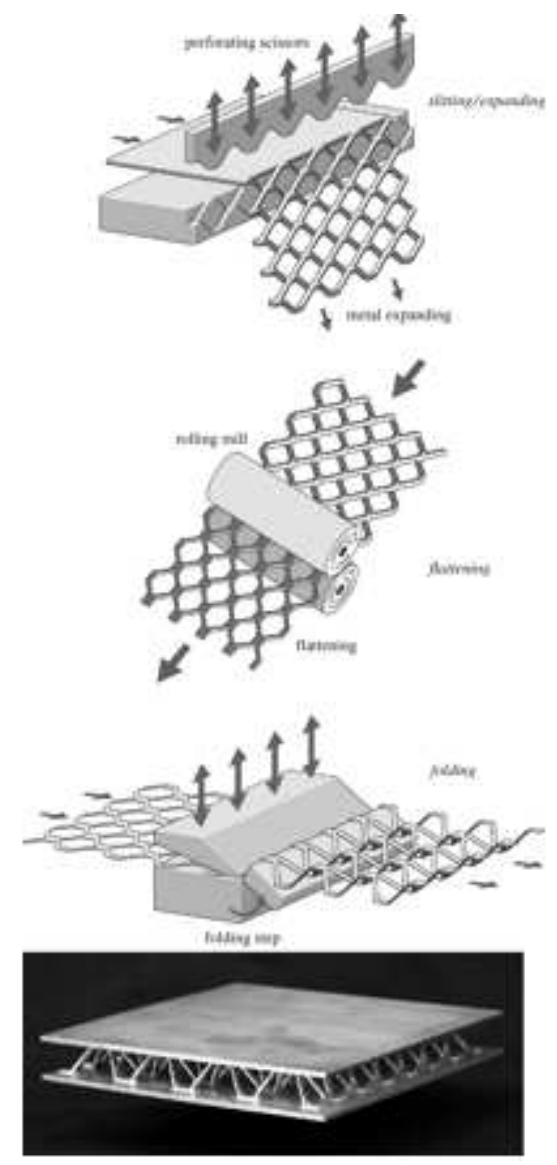

Figure 1: Production of pyramidal lattice core using perforation/rolling/folding technique on metals (From [3])

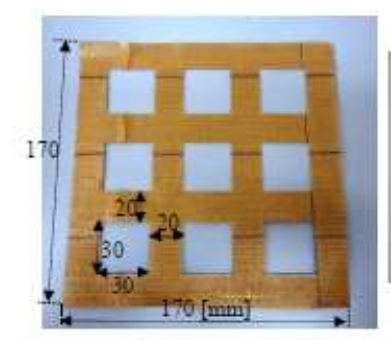

(a)

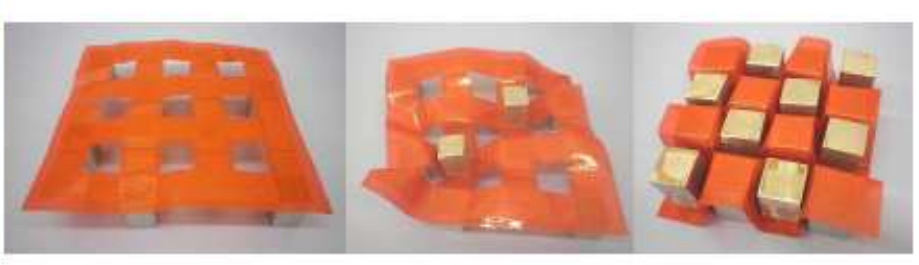

(b)

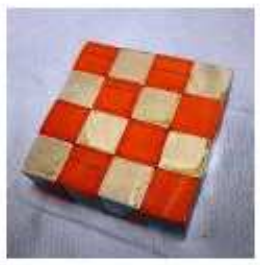

(c)

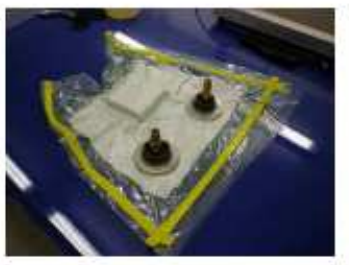

(d)

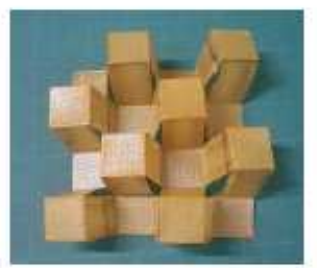

(e)

Figure 2: Steps of the manufacturing for the Kirigami auxetic pyramidal lattice core 


\section{METHODS}

\section{A. Mechanical homogenisation}

The equivalent mechanical properties of the Kirigami lattice structure have been computed using Finite-Element based homogenisation techniques for periodic media [18]. In a periodic medium, the generalised nodal displacement vector $\mathbf{u}$ can be expressed as $\mathbf{u}=\bar{\epsilon} \mathbf{x}+\mathbf{u}^{\prime}$, where $\bar{\epsilon}$ is an applied strain field, and $\mathbf{u}^{\prime}$ a slowly fluctuating periodic function of $\mathbf{u}$. The variational fluctuation to identify $\mathbf{u}^{\prime}$ is expressed by [18]:

$$
\left\{\mathbf{v}^{\prime}\right\}^{T}[\mathbf{K}]\left\{\mathbf{u}^{\prime}\right\}=\left\{\mathbf{v}^{\prime}\right\}^{T}\{\mathbf{F}\}
$$

Where $\left\{\mathbf{v}^{\prime}\right\}$ is another periodic fluctuating function solving Equation (1), $[\mathbf{K}]$ is the stiffness matrix of the system and $\{\mathbf{F}\}$ is a vector of generalised nodal forces. According to standard FE techniques, the stiffness matrix is defined as:

$$
[\mathbf{K}]=\sum_{e}[\mathbf{k}]_{e}=\frac{1}{V} \int_{e}[\mathbf{B}]^{T}[\mathbf{C}][\mathbf{B}] d V
$$

In (2), $[\mathbf{B}]$ contains the spatial derivatives of the FE shape functions, $[\mathbf{C}]$ the second order stress-strain tensor of the material associated to the $e^{\text {th }}$ element, and $V$ the volume of the overall model. When a prescribe strain field $\{\bar{\epsilon}\}$ is imposed, the generalised force vector $\{\mathbf{F}\}$ can be expressed as:

$$
\{\mathbf{F}\}=-[\overline{\mathbf{K}}]\{\bar{\epsilon}\}
$$

Where:

$$
[\overline{\mathbf{K}}]=\sum_{e}[\overline{\mathbf{k}}]_{e}=\frac{1}{V} \int_{e}[\mathbf{B}]^{T}[\mathbf{C}] d V
$$

Inserting (4) and (3) in (1) one obtains the homogenised stress-strain tensor relationship representing the equivalent material enclosed in the volume $V$ :

$$
[\overline{\mathbf{C}}]\{\bar{\epsilon}\}=\{\bar{\sigma}\}
$$

Where $\{\bar{\sigma}\}$ is the volume average vector of the stresses $\sigma_{i j}, i, j=1 \ldots 6$ for the representative unit cell of the periodic microstructure. The homogenised compliance matrix 
$[\overline{\mathbf{S}}]=[\overline{\mathbf{C}}]^{-\mathbf{1}}$ is equal, for a transverse isotropic material as:

$$
[\overline{\mathbf{S}}]=\left[\begin{array}{cccccc}
\frac{1}{E_{x}} & -\frac{\nu_{y x}}{E_{x}} & -\frac{\nu_{z x}}{E_{x}} & 0 & 0 & 0 \\
-\frac{\nu_{x y}}{E_{y}} & \frac{1}{E_{y}} & -\frac{\nu_{z y}}{E_{y}} & 0 & 0 & 0 \\
-\frac{\nu_{x z}}{E_{z}} & -\frac{\nu_{y z}}{E_{z}} & -\frac{1}{E_{z}} & 0 & 0 & 0 \\
0 & 0 & 0 & -\frac{1}{G_{x z}} & 0 & 0 \\
0 & 0 & 0 & 0 & \frac{1}{G_{y z}} & 0 \\
0 & 0 & 0 & 0 & 0 & \frac{1}{G_{x y}}
\end{array}\right]
$$

The representative unit element (RVE) cell used for the Kirigami pyramidal lattice is shown in Figure (3a). The pyramidal lattice is described using the nondimensional parameters $\alpha=a / b, \beta=t / l, \delta=l / b$ and internal cell angle $\theta$. An isotropic in-plane mechanical configuration is achieved for $\alpha=1$. The parameter $\delta$ indicates how far the behaviour of the ligament $l$ departs from a beam-like structure. Within the context of this work, only inplane isotropic configurations $(\alpha=1)$ have been considered. The FE model of the unit cell has been developed using the commercial code ANSYS 11.0 (Figure 3b). Three-dimensional structural hexahedral elements with Lagrangian interpolation functions SOLID45 (8 nodes and 3 DOFs) have been used to represent the pyramidal structure, with a uniform mesh size equivalent to $t / 2$ assumed after a mesh convergence test. The periodic boundary conditions [19] have been applied to the edges $x y$ on the unit cell, while the minimum and maximum $z$ coordinates have been kept free of imposed displacements corresponding to uniform strains, as to represent an infinite periodic core material for sandwich structures, having therefore a

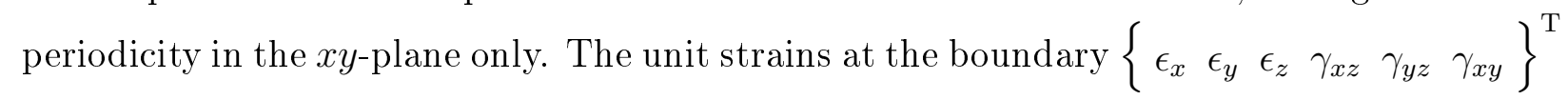
have been imposed as displacement fields, leading to a static linear problem solved with a Newton-Raphson solver. Depending on the specific combination of nondimensional geometry parameters used, the models had sizes ranging between 4296 to 12240 DOFs. The base mechanical properties of the core were representative of Kevlar 49/914 woven fabric [16], with Young's modulus $E_{c}=30 \mathrm{GPa}$, Poisson's ratio $\nu_{c}=0.4$ and density $\rho_{c}=1600 \mathrm{~kg} \mathrm{~m}^{-3}$. For all the simulations, the length of the ribs $l$ was assume constant at $10 \mathrm{~mm}$, while the relative density $\beta$ was imposed equal to $5 \%$ to avoid shear deformation contributions from the cross-section of the ribs, and assume thin beam-like or plate-like structures being present in the Kirigami lattice (i.e., consistent to samples produced with the manufacturing process shown in Figure 2). 


\section{B. Christoffel's equations}

The slowness curves have been simulated from the solution of Christoffel's equations. For a given $\mathbf{k}$ propagation direction, the equations have the corresponding eigenvalue problem $[20,21]$ :

$$
[\mathbf{\Gamma}]\{\mathbf{A}\}=\gamma\{\mathbf{A}\}
$$

Where $\boldsymbol{\Gamma}=k_{i} \bar{c}_{i j k l} k_{l}$ and $\gamma=\rho v^{2}$, while $\bar{c}_{i j k l}$ indicates the homogenised fourth-order stressstrain tensor of the equivalent material of the pyramidal auxetic lattice. The determinant of (7) equated to zero yields three eigenvalues in terms of $\rho v^{2}(\mathbf{k})$, leading to three slowness curves $s(\mathbf{k})=1 / v(\mathbf{k})$ associated to $|\mathbf{k}|=1$ [21]. Considering the wave vector $\mathbf{k}$ propagating in the $x z$ plane (with $\mathbf{k}=\cos (\phi) \mathbf{k}+\sin (\phi) \mathbf{i}, \mathbf{i}, \mathbf{k}$ being the unit versors of the $x$ and $z$ axis respectively), the matrix $\Gamma$ in (7) can be recast for the transverse isotropic microstructure as [21]:

$$
[\boldsymbol{\Gamma}]=\left[\begin{array}{ccc}
\bar{c}_{55}+\left(\bar{c}_{11}-\bar{c}_{55}\right) \sin ^{2} \phi & 0 & \left(\bar{c}_{13}+\bar{c}_{55}\right) \cos \phi \sin \phi \\
0 & \bar{c}_{44}+\left(\bar{c}_{66}-\bar{c}_{44}\right) \sin ^{2} \phi & 0 \\
\left(\bar{c}_{13}+\bar{c}_{55}\right) \cos \phi \sin \phi & 0 & \bar{c}_{33}+\left(\bar{c}_{55}-\bar{c}_{33}\right) \sin ^{2} \phi
\end{array}\right]
$$

When the elastic constants are normalised against $\bar{c}_{11} / \sqrt{\rho}$ ( $\rho$ being the density of the homogenised material), the three eigenvalues $\gamma_{i}$ give the slowness curves $s_{i}(\phi)=v_{i}^{-1}(\phi)=$ $\gamma_{i}^{-1 / 2}(\phi)$ in the $x z$ plane.

For wave propagation in the $x y$ plane (i.e., $\mathbf{k}=\cos (\phi) \mathbf{j}+\sin (\phi) \mathbf{i}$, with $\mathbf{i}, \mathbf{j}$ the unit versors along the $x$ and $y$ directions respectively), the $\boldsymbol{\Gamma}$ matrix assumes the following form [20]:

$$
[\boldsymbol{\Gamma}]=\left[\begin{array}{ccc}
\bar{c}_{11} \cos ^{2} \phi+\bar{c}_{66} \sin ^{2} \phi & \left(\bar{c}_{12}+\bar{c}_{66}\right) \cos \phi \sin \phi & 0 \\
\left(\bar{c}_{12}+\bar{c}_{66}\right) \cos \phi \sin \phi & \bar{c}_{66} \cos ^{2} \phi+\bar{c}_{22} \sin ^{2} \phi & 0 \\
0 & 0 & \bar{c}_{55} \cos ^{2} \phi+\bar{c}_{44} \sin ^{2} \phi
\end{array}\right]
$$

The slowness curves $s_{i}(\phi)=\gamma_{i}^{-1 / 2}(\phi)$ can also be calculated normalising the coefficients in (9) by $\bar{c}_{11} / \sqrt{\rho}$. The eigenvalues of (7) have been calculated using the det MatlabC routine, with the tensor coefficients $\bar{c}_{i j}$ obtained from the FE homogenisation procedure described above. 


\section{Floquet and Bloch theorems for elasto-dynamic dispersion analysis}

\section{Mathematical framework}

Let us consider an infinite periodic elastodynamic problem as presented in figure 4 . The harmonic homogeneous dynamical equilibrium of system is driven by the following partial derivative equation:

$$
\rho(\boldsymbol{x}) \omega^{2} \boldsymbol{w}(\boldsymbol{x})+\nabla \boldsymbol{C}(\boldsymbol{x}) \nabla_{\text {sym }}(\boldsymbol{w}(\boldsymbol{x}))=0 \forall \boldsymbol{x} \in \mathrm{R}^{3}
$$

where $\boldsymbol{w}(\boldsymbol{x})$ in $\mathrm{R}^{3}$ is the displacement vector, $\boldsymbol{C}(\boldsymbol{x})$ stands for the Hook elasticity tensor and $\varepsilon(\boldsymbol{x})=\nabla_{\text {sym }}(\boldsymbol{w}(\boldsymbol{x}))=\frac{1}{2}\left(\nabla \boldsymbol{w}^{T}(\boldsymbol{x})+\boldsymbol{w}(\boldsymbol{x}) \nabla^{T}\right)$ is the strain tensor. By considering a primitive cell of the periodic problem $\Omega_{R}$ and by using the Bloch theorem, the associated Bloch eigenmodes and the dispersion functions can be found by searching the eigen solutions of the homogeneous problem:

$$
\boldsymbol{w}(\boldsymbol{x})=\boldsymbol{w}_{n, k}(\boldsymbol{x}, \boldsymbol{k}) e^{i \boldsymbol{k} \cdot \boldsymbol{x}}
$$

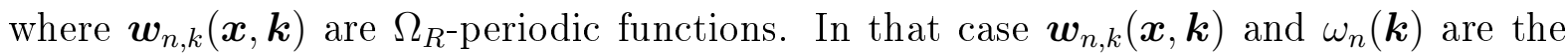
solutions of the following generalized eigenvalues problem of the shifted cell operator as described in:

$$
\begin{gathered}
\rho(\boldsymbol{x}) \omega_{n}(\boldsymbol{k})^{2} \boldsymbol{w}_{n, k}(\boldsymbol{x})+\nabla \boldsymbol{C}(\boldsymbol{x}) \nabla_{s y m}\left(\boldsymbol{w}_{n, k}(\boldsymbol{x})\right) \\
-i \boldsymbol{C}(\boldsymbol{x}) \nabla_{s y m}\left(\boldsymbol{w}_{n, k}(\boldsymbol{x})\right) \cdot \boldsymbol{k}-i \nabla \boldsymbol{C}(\boldsymbol{x}) \frac{1}{2}\left(\boldsymbol{w}_{n, k}(\boldsymbol{x}) \cdot \boldsymbol{k}^{T}+\boldsymbol{k} \cdot \boldsymbol{w}_{n, k}^{T}(\boldsymbol{x})\right) \\
+\boldsymbol{C}(\boldsymbol{x}) \frac{1}{2}\left(\boldsymbol{w}_{n, k}(\boldsymbol{x}) \cdot \boldsymbol{k}^{T}+\boldsymbol{k} \cdot \boldsymbol{w}_{n, k}^{T}(\boldsymbol{x})\right) \cdot \boldsymbol{k}=0 \\
\boldsymbol{w}_{n, k}(\boldsymbol{x}-\boldsymbol{R} \cdot \boldsymbol{n})-\boldsymbol{w}_{n, k}(\boldsymbol{x})=0 \forall \boldsymbol{x} \in \Gamma_{R}
\end{gathered}
$$

Let us consider the partial derivative equations (12) on a unit cell $\Omega_{R}$. That expression stands for a generalized eigenvalue problem leading to computation of the dispersion curves $\omega_{n}(\boldsymbol{k})$ and corresponding Floquet eigenvectors $\boldsymbol{w}_{n, k}(\boldsymbol{x})$. For 3D applications, the wave vectors are supposed to be complex if damping terms are added into equation (12), and can be therefore written as $\boldsymbol{k}=k\left[\begin{array}{c}\sin (\theta) \cos (\phi) \\ \sin (\theta) \sin (\phi) \\ \cos (\theta)\end{array}\right]=k \boldsymbol{\Phi}$, where $\theta$ and $\phi$ represent the direction 
angles into the reciprocal lattice domain, and $\boldsymbol{\Phi}$ as the direction vector. This decomposition assumes that real and imaginary parts of the vector $\boldsymbol{k}$ are co-linear.

After taking into consideration the periodicity conditions and a few arrangements, a weak formulation of the problem can be written as:

$$
\begin{gathered}
\forall \tilde{\boldsymbol{w}}_{n, k}(\boldsymbol{x}) \in\left\{H_{1}\left(\Omega_{R}, \mathrm{C}^{3}\right) / \tilde{\boldsymbol{w}}_{n, k}(\boldsymbol{x}-R \boldsymbol{n})=\tilde{\boldsymbol{w}}_{n, k}(\boldsymbol{x}) \forall \boldsymbol{x} \in \Gamma_{R}\right\} \\
\int_{\Omega_{R}} \rho(\boldsymbol{x}) \omega_{n}^{2}(\boldsymbol{k}) \tilde{\boldsymbol{w}}_{n, k}(\boldsymbol{x}) \boldsymbol{w}_{n, k}(\boldsymbol{x})-\tilde{\boldsymbol{\varepsilon}}_{n, k}(\boldsymbol{x}) \boldsymbol{C}(\boldsymbol{x}) \boldsymbol{\varepsilon}_{n, k}(\boldsymbol{x}) \\
+i k \tilde{\boldsymbol{\kappa}}_{n, k}(\boldsymbol{x}) \boldsymbol{C}(\boldsymbol{x}), \boldsymbol{\varepsilon}_{n, k}(\boldsymbol{x})-i k \tilde{\boldsymbol{\varepsilon}}_{n, k}(\boldsymbol{x}) \boldsymbol{C}(\boldsymbol{x}) \boldsymbol{\kappa}_{n, k}(\boldsymbol{x}) \\
+k^{2} \tilde{\boldsymbol{\kappa}}_{n, k}(\boldsymbol{x}) \boldsymbol{C}(\boldsymbol{x}) \boldsymbol{\kappa}_{n, k}(\boldsymbol{x}) d \Omega=0
\end{gathered}
$$

The numerical implementation is obtained by using a standard finite elements method to discretise the weak formulation (14) . The assembled matrix equation is given by:

$$
\left.(\boldsymbol{K}+\lambda \boldsymbol{L}\}(\boldsymbol{\Phi})-\lambda^{2} \boldsymbol{H}(\{\boldsymbol{\Phi}\})-\omega_{n}^{2}(\lambda,(\boldsymbol{\Phi}\}) \boldsymbol{M}\right) \boldsymbol{w}_{n, k}(\boldsymbol{\Phi})=0
$$

where $\lambda=i k, \boldsymbol{M}$ and $\boldsymbol{K}$ are the standard symmetric definite mass and symmetric semidefinite stiffness matrices respectively, $\boldsymbol{L}$ is a skew-symmetric matrix and $\boldsymbol{H}$ is a symmetric semi-definite positive matrix:

$$
\begin{gathered}
\boldsymbol{M} \rightarrow \int_{\Omega_{R}} \rho(\boldsymbol{x}) \omega_{n}^{2}(\boldsymbol{k}) \tilde{\boldsymbol{w}}_{n, k}(\boldsymbol{x}) \boldsymbol{w}_{n, k}(\boldsymbol{x}) d \Omega \\
\boldsymbol{K} \rightarrow \int_{\Omega_{R}} \tilde{\boldsymbol{\varepsilon}}_{n, k}(\boldsymbol{x}) \boldsymbol{C}(\boldsymbol{x}) \boldsymbol{\varepsilon}_{n, k}(\boldsymbol{x}) d \Omega \\
\boldsymbol{L} \rightarrow \int_{\Omega_{R}}-\tilde{\boldsymbol{\kappa}}_{n, k}(\boldsymbol{x}) \boldsymbol{C}(\boldsymbol{x}) \boldsymbol{\varepsilon}_{n, k}(\boldsymbol{x})+\tilde{\boldsymbol{\varepsilon}}_{n, k}(\boldsymbol{x}) \boldsymbol{C}(\boldsymbol{x}) \boldsymbol{\kappa}_{n, k}(\boldsymbol{x}) d \Omega \\
\boldsymbol{H} \rightarrow \int_{\Omega_{R}} \tilde{\boldsymbol{\kappa}}_{n, k}(\boldsymbol{x}) \boldsymbol{C}(\boldsymbol{x}) \boldsymbol{\kappa}_{n, k}(\boldsymbol{x}) d \Omega
\end{gathered}
$$

When $k$ and $\boldsymbol{\Phi}$ are fixed, the system (15) is a linear eigen value problem, the solution of which allows computing the dispersion functions $\omega_{n}^{2}(\boldsymbol{k}, \boldsymbol{\Phi})$ and the associated Bloch eigenvector $\boldsymbol{w}_{n, k}(\boldsymbol{\Phi})$.

This approach is classically used for homogenization, spectral asymptotic analysis [22]. In the context of wave propagation, dispersion characteristics can be derived even if Floquet propagators are preferred for $1 \mathrm{D}$ or quasi 1D computation, as indicated by [23], [24] or [25]. Most of the time, these techniques are applied for undamped mechanical systems, represented by a set of constant and real matrices. The classical approach consists in using a mesh of the (real) $k$-space (i.e $k$ or $\lambda$ and $\boldsymbol{\Phi}$ ) inside the first Brillouin zone for obtaining the corresponding frequency dispersion diagrams and the associated Floquet vectors. For 
undamped systems, only propagating or evanescent waves exist, corresponding to families of eigensolutions purely real or imaginary. Discrimination between each class of waves is quite straightforward. If a damped system is considered, i.e. if matrices $\boldsymbol{K}, \boldsymbol{L}, \boldsymbol{H}$ are complex, the evanescent part of the propagating waves appear as the imaginary part of $\omega_{n}^{2}(\lambda, \boldsymbol{\Phi})$ and vice versa. It then becomes very difficult to distinguish the two families of waves, but also to compute the corresponding physical wave's movements by applying spatial deconvolution.

It is possible to rearrange the system in order to take into account damping effects [26]. This formulation also provides suitable contents for time/space deconvolution and for the computation of diffusion properties as defined by [27] or [24]. It consists in considering the following generalised eigenvalue problem:

$$
\left.\left(\boldsymbol{K}-\omega^{2} \boldsymbol{M}\right)+\lambda_{n}(\omega, \boldsymbol{\Phi}) \boldsymbol{L}(\boldsymbol{\Phi})-\lambda_{n}^{2}(\omega, \boldsymbol{\Phi}) \boldsymbol{H}(\boldsymbol{\Phi})\right) \boldsymbol{w}_{n, k}(\boldsymbol{\Phi})=0
$$

In this problem, the pulsation $\omega$ and the propagative angle $\boldsymbol{\Phi}$ are fixed real parameters. Wave's numbers $\lambda_{n}=i k_{n}$ and associated Floquet vectors $\boldsymbol{w}_{n, k}$ are then computed by solving the quadratic eigen problem (17). This approach allows to introduce frequency-dependent matrices corresponding to generalized damping terms (viscoelasticity), multiphysics coupling (electromechanical with electronic ordinary differential equation), foam (Biot-Allard model) or open domain boundary conditions (Sommerfeld condition). Based on this approach, an inverse Fourier transformation in the $k$-space domain leads to evaluate the physical wave's displacements and energy diffusion operator when the periodic distribution is connected to another system [27]. Another temporal inverse Fourier transformation can provide a way to access spacial-temporal response for non-homogeneous initial conditions. As $\boldsymbol{L}$ is skewsymmetric, the resulting eigenvalues are quadruple $(\lambda, \bar{\lambda},-\lambda,-\bar{\lambda})$, and collapsing into real or imaginary pairs (or a single zero) when all matrices are real for undamped systems. In the general damped case, one obtains a pair of real eigenvalues corresponding to evanescent modes oriented in two opposite directions on the $k$-space, and imaginary values to two traveling waves propagating in opposite direction. The eigensolutions are similar to those given by the SAFE method in 1D for homogeneous material (non periodic), and additional important properties can be extrapolated from [28]. The validity of the method proposed in this work has been proved with several test cases in [26].

As previously mentioned, the real part of $\boldsymbol{k}=k \boldsymbol{\Phi}$ vector is classically restricted to the first Brillouin zone. In the quadratic eigen value problem (17) the validity of the computed 
solution is no longer confined to a specific Brillouin zone. For a direction vector $\boldsymbol{\Phi}$ orthogonal to the lattice facets (i.e. for $\boldsymbol{\Phi}_{p 1}=[1,0]^{T}$ and $\boldsymbol{\Phi}_{p 2}=[0,1]^{T}$ in a $2 \mathrm{D}$ rectangular cell), the periodical conditions expressed for one dimensional waveguide are still valid: if $\lambda_{j}\left(\boldsymbol{\Phi}_{p}\right)$ is an eigen value associated to $\boldsymbol{w}_{j, k}\left(\boldsymbol{\Phi}_{p}\right)$, then $\forall \boldsymbol{m} \in \mathrm{Z}^{3}, \lambda+i . \boldsymbol{\Phi}_{p}^{T}(\boldsymbol{G} \cdot \boldsymbol{m})$ is also an eigenvalue associated to $\boldsymbol{w}_{j, k}\left(\boldsymbol{\Phi}_{p}\right) e^{-i . \boldsymbol{\Phi}_{p}^{T}(\boldsymbol{G} \cdot \boldsymbol{m}) x}$. Thus, for undamped systems, all the eigenvalues are periodically distributed in the $k$-space along its principal directions.

\section{Computation of evanescence and damped power flow criteria}

One aim of this work is to provide a numerical methodology to describe the particular behavior of the energy flow into the periodically Kirigami auxetic core. One therefore needs to define suitable indicator for distinguishing propagative and evanescent behavior especially when damped system is concerned. The capability of a given Bloch wave to transport energy is given by its group velocity, which indicates how energy is transported into the considered system, allowing also to distinguish between propagative and evanescent waves. If a Bloch eigen solution (i.e $\left.\boldsymbol{u}_{n}(\omega, \phi), \boldsymbol{k}_{n}(\omega)\right)$ is considered, the associated group velocity vector [29] is given by:

$$
C_{g_{n}}(\omega, \phi)=\nabla_{\boldsymbol{k}} \omega=\frac{\langle\langle\boldsymbol{S}\rangle\rangle}{\left\langle\left\langle e_{t o t}\right\rangle\right\rangle}=\frac{\langle\boldsymbol{I}\rangle}{\left\langle E_{t o t}\right\rangle}
$$

Where $\langle\langle:\rangle\rangle$ is the spatial and time average respectively on one cell and one period of time, is the density of energy flow, $\boldsymbol{I}$ the mean intensity and $e_{t o t}, E_{t o t}$ the total energy and its time average on a period (see \cite\{Maysenholder1994\} for details).

The intensity vector $\boldsymbol{I}$ is expressed as:

$$
\left\langle\boldsymbol{I}_{n}\right\rangle=-\frac{\omega}{2} R e\left(\int_{\Omega_{x}} C\left(\varepsilon_{n}(\boldsymbol{x})+i k \Xi_{n}(\boldsymbol{x})\right) \cdot\left(\boldsymbol{w}_{n}^{*}(\boldsymbol{x})\right) \frac{d \Omega}{V_{o l}}\right)
$$

Where .* is the complex conjugate, $R e$ stands for real part and $V_{o l}$ for the do-

main volume. As the spacial-temporal average of the system Lagragian is null (see $\backslash$ cite\{Maysenholder1994\}), the total energy average is approximated by only computing the kinetic energy average:

$$
\left\langle E_{t o t}\right\rangle=\frac{1}{2 V_{o l}}\left(\int_{\Omega_{x}} \rho \omega^{2} \boldsymbol{w}_{n}(\boldsymbol{x}, \omega, \phi) \cdot \boldsymbol{w}_{n}^{*}(\boldsymbol{x}, \omega, \phi) d \Omega\right)
$$


The group velocity vectors $C_{g_{n}}(\omega, \phi)$ are computed for all wave numbers at each frequency.

\section{Test case for the Kirigami pyramidal lattice}

The proposed methodology is used to study the wave dispersion into a 2 dimensional Kirigami auxetic lattice. It consists of an infinite periodic bi-dimensional waveguide made of a periodic distribution of the unitary cell presented in figure 5. The system is made of a $2.5 \mathrm{~mm}$ thick plate assembly made of isotropic damped non-woven Kevlar polymer with the same mechanical characteristics used for the FE homogenisation. To consider the intrinsic material damping of the core material, an hysteretic damping factor of 0.001 has been assumed, consistent to hysteretic loss factors of thermoset/epoxy prepregs [30]. The cell size is $120 \mathrm{~mm}^{2}$. The method allows us to compute eigen frequencies corresponding to any $\boldsymbol{k}$ vector described in cylindrical coordinates system by its radius $k$ and its angle $\phi$ in the whole first Brillouin domain. The numerical implementation is based on the 3D weak formulation (14), using a bi-dimensional orientation in the $k$-space by imposing $\{\boldsymbol{\Phi}\}=\left[\begin{array}{c}\cos (\phi) \\ \sin (\phi) \\ 0\end{array}\right]$. The applied boundary conditions are equalities of all 3D displacements on the two pairs of lateral faces $\Gamma_{r_{1}^{+}} \Gamma_{r_{1}^{-}}$and $\Gamma_{r_{2}^{+}} \Gamma_{r_{2}^{-}}$on all cell boundaries. To impose such Dirichlet boundary conditions an extrusion coupling variable maps of displacements from the source face is exported to the destination face corresponding to the opposite one (i.e., from $\Gamma_{r_{1}^{+}} t o \Gamma_{r_{1}^{-}}$). As the domains are of the same space dimension, we typically use a point-wise mapping. The exported mapping is also coupled to the destination displacement by using dedicated Lagrange multipliers. The implementation is made with COMSOL Multiphysics(C) platform and parametric computation to obtain $k(\omega, \phi)$ is carried out with Matlab(C) routines. For each parameters $\omega$ and $\phi$, the quadratic eigenvalue problem can be reformulated as a first order one by doubling the state dimension. After constraint handling, it is possible to write the system in the form $\{\boldsymbol{A}\}\{\boldsymbol{x}\}=\lambda\{\boldsymbol{B}\}\{\boldsymbol{x}\}$. The algorithm computes the largest eigenvalues of the matrix $\{\boldsymbol{C}\}=\{\boldsymbol{A}\}^{-1}\{\boldsymbol{B}\}$. To do this, the solver uses the ARPACK FORTRAN routines for large-scale eigenvalue problems which is described by [31]. This code is based on a variant of the Arnoldi algorithm: the implicitly restarted Arnoldi method (IRAM). The ARPACK routines must perform several matrix-vector multiplications $\{\boldsymbol{C}\}\{\boldsymbol{v}\}$, which 
are accomplished here by solving the linear system $\{\boldsymbol{A}\}\{\boldsymbol{x}\}=\{\boldsymbol{B}\}\{\boldsymbol{v}\}$ using the PARDISO solver developed by [32]. This procedure uses double precision floating point numbers and is implemented using out of core memory management in order to avoid any memory problem even when dense (and converged) mesh is considered (Figure 6). The first mesh case consists of 4478 tetrahedral Lagrange quadratic elements for 25938 degrees of freedom and the refined one of 40729 tetrahedral Lagrange quadratic elements for 197430 degrees of freedom. In the test cases considered within this work, the frequency range is $\omega=2 \pi .[1000: 1000: 10000]$ (between $1 \mathrm{kHz}$ and $10 \mathrm{kHz}$ ) and $\phi=\left[0: \frac{\pi}{20}: \frac{\pi}{2}\right]$.

\section{RESULTS}

\section{A. Mechanical properties}

Figure 7a shows the variation of the in-plane Poisson's ratio $\nu_{x y}$ (equal to $\nu_{y x}$ for symmetry reasons) for different $\delta$ values at varying internal cell angles $(\beta=0.05)$. The auxetic (negative Poisson's ratio) behaviour of the Kirigami lattice is evident for the $\theta$ range considered, apart from very small $\left(\theta<2^{\circ}\right)$ and large $\left(\theta>78^{\circ}\right)$. Small angles indicate configurations where the ligaments $l$ tend to be almost flat (horizontal), while high angles are related to configurations where the ligaments tend to stand upright (vertical). In both cases, the ligaments $l$ tend to be dominated by axial deformation mechanisms, which contribute significantly to a lowering on the Poisson's ratio values in centresymmetric honeycomb assemblies with ribs having flexural behaviour [33]. Between $10^{\circ}$ and $30^{\circ}$ the Poisson's ratio tends to have its highest magnitude $(\sim-0.9$ for $\delta=5.0)$, which tends to decrease as soon as the stretching effects of the ligaments start to be present in the microstructure. The more the ligament also assumes a plate-like shape (i.e., lowering $\delta$ values), the lower the magnitude of the in-plane Poisson's ratio, with the minimum value now reached at lower internal cell angle $\left(\nu_{x y}=-0.6\right.$ for $\delta=1.0$ ). On the contrary, the out-of-plane Poisson's ratio $\nu_{x z}$ (Figure $7 \mathrm{~b}$ ) is always positive, ranging between 0.45 and 0.2 , with a semi-monotonic decrease with the internal cell angle, more pronounced with plate-like ribs $l(\delta=1.0)$.

Figure $8 \mathrm{a}$ shows the variation of the in-plane $\left(E_{x}=E_{y}\right)$ and out-of-plane $\left(E_{z}\right)$ Young's modulus versus the internal cell angle for two rib aspect ratios $(\delta=1.0$ and $\delta=3.0)$. An important aspect to highlight is the fact that the transverse stiffness is lower than in the 
in-plane one. For very small angles (inset diagram of Figure 8 a) the ratio $E_{x} / E_{z}$ is 2 for $\delta=1.0$, while for $\theta>20^{\circ}$ the ratio can vary between 4 and 9 times. Lower stiffness ratios are observed for increasing $\delta$ values. This behaviour is unusual for classical cellular structures, who show a linear scaling versus $\beta$ of the transverse mechanical properties, while the inplane ones scale with $\beta^{3}$ [34]. However, the Kirigami pyramidal lattice can be thought as a prismatic honeycomb (half) section, where the ribs deform under bending, while the $x y$-plane properties are dominated by membrane and shear deformations of the ribs $l$. In that sense, the pyramidal lattice considered in this work behaves like a centresymmetric honeycomb with its prismatic cross-section used as through-the-thickness reinforcement. A even more pronounced ratio between in-plane and out-of-plane shear can be observed in Figure 8b, where $G_{x y} / G_{x z}$ ratios up to 60 can be observed for $\theta<3^{\circ}$ and $\delta=1.0$. Interestingly, for both $\delta$ configurations one can observe a transverse shear stiffness inversion above $\theta=55^{\circ}$, where the out-of-plane shear modulus becomes higher than the in-plane one. This behavior is likely to be conducted to increasing interaction between the membrane stiffness within the ribs $l$ and the bending one in the plates $b$ at the base of the unit cell (Figure 1a), which tends to equalise for a specific cell angle and $\delta$ value at constant $\alpha$.

\section{B. Slowness curves}

Figure 9 shows the slowness curves in the $x z$ plane (equal for symmetry in the $y z$ plane) for $\delta=1.0$ (Figure $9 \mathrm{a}$ ) and $\delta=5.0$ (Figure 9b). At a constant internal cell angle $(\theta=$ $\left.20^{\circ}\right)$, the third slowness curve $s_{3}(\phi)$ shows a very strong directivity at $\phi=0, \pi / 2, \pi, 3 \pi / 2$ (corresponding also to the main axis of the ribs $l$ ). The first two slowness curves $s_{1}(\phi)$ and $s_{2}(\phi)$ have however a more interesting behaviour, with a near zero-slope between $55^{\circ}<$ $\phi<135^{\circ}$. The zero-slowness is lower in terms of range for the second slowness curve, and depends on the rib aspect ratio $\delta$. While a $10 \%$ difference in terms of angle $\phi$ range for the zero-slope exists for the lattice with $\delta=1.0$, the $s_{1}(\phi)$ curve shows a flat slope extending almost between $20^{\circ}<\phi<160^{\circ}$ for ribs $l$ assuming a more beam-like structure $(\delta=5.0)$. In normalised terms, the second slowness curve in $\delta=1.0$ corresponds to higher velocities $v$ (solutions of the Christoffel's equation (7)) than the ones for the lattice configuration with $\delta=5.0$. The existence of zero-slope slowness curves in centersymmetric monoclinic lattices has been suggested as a possible mechanism to generate solitons along specific spatial 
directions on the microstructure [21]. In that sense, the Kirigami pyramidal auxetic lattice show some interesting zero-slope characteristics for the first and second slowness curves, although not for the first one as identified in specific monoclinic configurations by Wang and Ryne [21]. No zero-slope slowness curve can be observed for the wave propagation in the $x y$ plane, with an isotropic directivity for $s_{1}(\phi)$ and $s_{3}(\phi)$, and a strong directionality in $s_{2}(\phi)$ for $\phi=0, \pi / 2, \pi, 3 \pi / 2$ (Figure 10). As in the case of the $x z$ plane wave propagation, the velocities corresponding to the second slowness curve tend to be 2.5 times lower in normalised terms for the beam-like lattice ribs $(\delta=5.0)$, than the equal aspect ratio (and plate-like) configurations with $\delta=1.0$.

\section{Wave dispersion}

In this section, all quantities of interest are given in nondimensional units. The reference velocity is considered as:

$$
c_{T}=\sqrt{\frac{E_{c}}{2\left(1-\nu_{c}\right) \rho_{c}}}
$$

where $E_{c}, \nu_{c}$ and $\rho_{c}$ are the Young's modulus, Poisson's ratio and density of the base material, and the associated reference frequency is:

$$
f_{\text {ref }}=\frac{\pi c_{T}}{L}
$$

\section{Dispersion along $\Gamma-X$ direction of the system}

Figure 11 shows the real part of the dispersion curves (i.e $\operatorname{Re}\left(k_{n}\right)$ ) in the case of unrefined meshes, including all calculated waves number. The obtained results indicate a large complexity of the vibroacoustic behavior of the Kirigami pyramidal lattice system, incorporating numerous evanescent modes and complex dispersion curves for propagative ones. This last mode is evaluated by using energy velocity criterion 18 so that: $\left\{\boldsymbol{C}_{g}\right\}_{n}(\omega, \phi) .\{\boldsymbol{\Phi}\}>\tau c_{T}$ with $\tau=0.5 \%$. The retained propagative waves are presented in figure 12 . It is possible to evaluate the system frequency band gaps, with the first one between $1 \%$ and $1.7 \%$ of the reference frequency, the second between $2 \%$ and $2.7 \%$. A narrow band-gap one can be identified around $4 \% \mathrm{kHz}$, while the last one is present between $0.57 \%$ and $0.64 \%$. Standard Lamb waves modes $\left(A_{0}, S_{o}\right)$ can be observed in every low frequency band. The corre- 
sponding group velocities diagram is shown in figure 13. A clear band gap structure and particular propagative characteristics (likely attributed to the unusual auxetic behavior) can be observed between $0.6 \%$ and $1 \%$ of the reference frequency.

\section{Dispersion of the system in the whole bi-dimensional k-space}

The proposed computational method allows us to compute multi modal wave's propagation in the complete bi-dimensional $k$-space of the first Brillouin zone. The proposed methodology is based on the computation of frequency-dependent complex wave numbers. The Bloch theorem is expanded in the case of damped systems and the results obtained become complex integrating phase velocity and evanescent part for each computed wave number associated to the real and imaginary parts of the obtained eigenvalues of equation. The damping behavior is introduced by assuming a complex Hook elasticity tensor. The same methodology could have been realized by introducing any kind of linear viscoelastic modeling such as viscous behavior or any other complex frequency-dependent terms.

Figure 14 illustrates the typical results of the analysis. Propagative wave numbers of the damped system are shown for different angles from $\phi=0$ to $\phi=45^{\circ}$ with a step of $9^{\circ}$. It can be observed that if $\phi=0$, the symmetry illustrated in figure 5 still exists, while as soon as other directions are considered it appears difficult to reshape the obtained results at the right location into the first Brillouin zone. This can be explained by the fact that the periodicity of the initial pattern is lost when the orientation is not parallel to one of the sides of the initial cell, and lacks a two dimensional consistent transformation. The observed band gap structure also depends onto the propagative angles. Therefore, to evaluate the band-gap of the periodic system, an indicator of minimal evanescence ratio of all the computed waves for each considered frequency can be used, defined as:

$$
\operatorname{Ind}(\omega, \phi)=\min _{n}\left|\frac{\operatorname{Real}\left(\lambda_{n}\right)}{\left|\left(\lambda_{n}\right)\right|}\right|
$$

Figure ?? shows the plot of this indicator in the whole $2 \mathrm{D}$ domain. The location of the band gaps can be observed clearly and indicate that specific directivity can exists at certain frequencies, as the band gaps are not axisymmetric and can be located between specific angles. Maximum evanescence is observed along the directions of the Kirigami pyramidal ribs of length $l$ for low frequency ranges (below $1 \mathrm{kHz}$ ). Between 2.0 and $2.5 \mathrm{kHz}$ the 
maximum evanescence shows an isotropic directivity, while below $7.5 \mathrm{kHz}$ the maximum directivity is again observed along the axis of the segments $l$ of the auxetic lattice.

\section{CONCLUSIONS}

The Kirigami pyramidal lattice described in this work has shown the following characteristics:

1. The lattice has an in-plane auxetic behaviour, with values of the Negative Poisson's ratio depending over the geometry parameters of the cellular configurations. Plate-like ribs tend to decrease the auxeticity of the 3D honeycomb. The out-of-plane Poisson's ratio is positive for all the configurations considered.

2. In-plane uniaxial stiffness (i.e., Young's modulus $E_{x}$ ) is higher compared to the transverse case. The Kirigami pyramidal lattice behaves like a prismatic honeycomb with the cell face oriented through the thickness, therefore explaining this rather peculiar mechanical behaviour. A similar trend is observed also for the shear modulus, although it is possible to identify a combination of internal cell angle and rib aspect ratio $\delta$ that leads to $G_{x y}=G_{x z}$ situation.

3. Zero-slope slowness curves can be observed for the wave propagation in the $x z$ plane, for the first and second curves respectively, suggesting possible directions for the wave vector where solitons could be created using this lattice as platform. No zero-curvature for the slowness curves has been identified in the $x y$ plane.

4. When considering a bi-dimensional wave propagation taking into account the effective scale of the microstructure (i.e., not being confined to the long wavelength approximation of points 1-3), the Kirigami lattice shows a complex wave pattern even in the $x y$ plane when a small values of hysteretic damping is considered. Using an evanescence index created for this lattice, it is possible to identify evanescence directivity patterns with directionality depending of the multi-modal characteristics of the Kirigami auxetic pyramidal lattice. 


\section{Acknowledgments}

This work has been partially supported by grant-in-aid for JSPS Fellows by the Japan Society for Promotion of Science.

[1] D. T. Queheillalt, H. N. Wadley, Pyramidal lattice truss structures with hollow trusses, Materials Science and Engineering: A 397 (2005) 132 - 137, ISSN 0921-5093, doi: \bibinfo\{doi $\}\{10$. 1016/j.msea.2005.02.048\}.

[2] D. T. Queheillalt, H. N. Wadley, Titanium alloy lattice truss structures, Materials \&amp; Design 30 (2009) 1966 - 1975, ISSN 0261-3069, doi: \bibinfo\{doi\}\{10.1016/j.matdes.2008.09. $015\}$.

[3] H. N. Wadley, Multifunctional periodic cellular metals, Philosophical Transactions of the Royal Society A: Mathematical, Physical and Engineering Sciences 364 (1838) (2006) 31-68, doi: $\backslash$ bibinfo $\{$ doi $\}\{10.1098 /$ rsta.2005.1697\} .

[4] S. M. Pingle, N. A. Fleck, V. S. Deshpande, H. N. G. Wadley, Collapse mechanism maps for a hollow pyramidal lattice, Proceedings of the Royal Society A: Mathematical, Physical and Engineering Science 467 (2128) (2011) 985-1011, doi: bibinfo\{doi\}\{10.1098/rspa.2010.0329\}.

[5] Alderson A, Alderson K L, Auxetic Materials, Proceedings of the Institution of Mechanical Engineers, Part G: Journal of Aerospace Engineering 221 (2007) 565 - 575.

[6] S. Gonella, M. Ruzzene, Analysis of in-plane wave propagation in hexagonal and re-entrant lattices, J. Sound Vibrat. 312 (2008) 125-139.

[7] Spadoni A, Ruzzene M, Numerical and Experimental Analysis of The Static Compliance of Chiral Truss Core Airfoils, Journal of Mechanics of Materials and Structures 2 (2007) 965 981.

[8] Tee K F, Spadoni A, Scarpa F, Ruzzene M, Wave propagation in auxetic tetrachiral honeycombs, ASME Journal of Vibration and Acoustics 132 (2010) 031007 - 031015.

[9] Martin J, Heyder-Bruckner JJ, Remillat C D L, Scarpa F, Potter K, Ruzzene M, The hexachiral prismatic wingbox concept, Physica Status Solidi B 245 (2008) $570-577$.

[10] Bettini P, Airoldi A, Sala G, Landro L D, Ruzzene M, Spadoni A, Composite chiral structures for morphing airfoils: Numerical analyses and development of a manufacturing process, 
Composites Part B: Engineering 41 (2010) 133 - 147.

[11] J. N. Grima, J. J. Williams, R. Gatt, K. E. Evans, Modelling of auxetic networked polymers built from calix[4]arene building blocks, Molecular Simulation 31 (13) (2005) 907-913, doi: $\backslash$ bibinfo $\{$ doi $\}\{10.1080 / 08927020500392114\}$.

[12] L. J. Hall, V. R. Coluci, D. S. GalvÃ£o, M. E. Kozlov, M. Zhang, S. O. Dantas, R. H. Baughman, Sign Change of Poisson's Ratio for Carbon Nanotube Sheets, Science 320 (5875) (2008) 504-507, doi: \bibinfo $\{$ doi $\}\{10.1126 /$ science.1149815\}.

[13] V. R. Coluci, L. J. Hall, M. E. Kozlov, M. Zhang, S. O. Dantas, D. S. Galvão, R. H. Baughman, Modeling the auxetic transition for carbon nanotube sheets, Phys. Rev. B 78 (2008) 115408, doi: \bibinfo $\{$ doi $\}\{10.1103 /$ PhysRevB.78.115408\}.

[14] T. Nojima, K. Saito, Development of Newly Designed Ultra-Light Core Structures, JSME International Journal Series A 49 (2006) 38-42.

[15] K. Saito, F. Scarpa, R. Neville, Origami Composite Auxetic Honeycomb, in: 16th International Conference on Composite Structures (ICCS16), 117, 2011.

[16] K. Saito, F. Agnese, F. Scarpa, A Cellular Kirigami Morphing Wingbox Concept, Journal of Intelligent Material Systems and Structures 22 (9) (2011) 935-944, doi: $\backslash$ bibinfo $\{$ doi $\}\{10.1177 /$ 1045389X11416030\}.

[17] J. P. Wolfe, Imaging Phonons, Cambridge University Press, Cambridge, UK., 1998.

[18] M. Bornert, T. Bretheau, P. Gilormini, Homogénéisation en mécanique des matériaux 1, HERMES Science Europe Ltd, 2001.

[19] G. Odegard, Constitutive modeling of piezoelectric polymer composites, Acta Materialia 52 (18) (2004) 5315 - 5330, ISSN 1359-6454, doi: \bibinfo\{doi\}\{10.1016/j.actamat.2004.07. $037\}$.

[20] A. H. Nayfeh, Wave propagation in layered anisotropic media with Applications to Composites, North Holland, Elsevier Science B.V., 1995.

[21] L. Wang, K. G. Ryne, Existence of extraordinary zero-curvature slowness curve in anisotropic elastic media, The Journal of the Acoustical Society of America 122 (4) (2007) 1873-1875, doi: $\backslash$ bibinfo $\{$ doi $\}\{10.1121 / 1.2770546\}$.

[22] G. Allaire, C. Congas, Bloch Waves Homogenization and Spectral Asymptotic Analysis, Journal de Mathématiques Pures et Appliquées 77 (1998) 153-208.

[23] M. N. Ichchou, S. Akrout, J. Mencik, Guided waves group and energy velocities via finite 
elements, Journal of Sound and Vibration 305 (4-5) (2007) 931-944, ISSN 0022-460X, doi: $\backslash$ bibinfo $\{$ doi $\}\{\{10.1016 /$ j.jsv.2007.05.007 $\}$.

[24] J. Mencik, M. Ichchou, Multi-mode propagation and diffusion in structures through finite elements, European Journal of Mechanics A-Solids 24 (5) (2005) 877-898, ISSN 0997-7538, doi: \bibinfo $\{$ doi $\}\{\{10.1016 /$ j.euromechsol.2005.05.004\}\}.

[25] L. Houillon, M. Ichchou, L. Jezequel, Wave motion in thin-walled structures, Journal of Sound and Vibration 281 (3-5) (2005) 483-507, ISSN 0022-460X, doi: $\backslash$ bibinfo $\{$ doi $\}\{\{10.1016 / j . j s v$. 2004.01.020\}\}.

[26] M. Collet, M. Ouisse, M. Ruzzene, M. Ichchou, A Floquet-Bloch decomposition of the elastodynamical equations: application to bi-dimensional wave's dispersion computation of damped mechanical system., International Journal of Solids and Structures 48 (2011) 2837-2848.

[27] M. Collet, K. Cunefare, N. Ichchou, Wave Motion Optimization in Periodically Distributed Shunted Piezocomposite Beam Structures, Journal of Int Mat Syst and Struct 20 (7) (2009) $787-808$.

[28] L. Gavrić, Computation of propagative waves in free rail using a finite element technique, Journal of Sound and Vibration 185 (1995) 531-543.

[29] W. Maysenhölder, Körperschall-energie Grundlagen zur Berechnung von Energiedichten und Intensitäten, Wissenschaftliche Verlagsgesellschaft, Stuttgart, 1994.

[30] J.-M. Berthelot, M. Assarar, Y. Sefrani, A. E. Mahi, Damping analysis of composite materials and structures, Composite Structures 85 (3) (2008) 189 - 204, ISSN 0263-8223, doi: $\backslash$ bibinfo $\{$ doi $\}\{10.1016 /$ j.compstruct.2007.10.024\}.

[31] R. Lehoucq, D. Sorensen, C. Yang, ARPACK users' guide: solution of large-scale eigenvalue problems with implicitly restarted Arnoldi methods, Siam, ISBN 0898714079, 1998.

[32] O. Schenk, K. Gärtner, Solving unsymmetric sparse systems of linear equations with PARDISO, Future Generation Computer Systems 20 (3) (2004) 475-487, ISSN 0167-739X.

[33] F. Scarpa, P. Panayiotou, G. Tomlinson, Numerical and experimental uniaxial loading on inplane auxetic honeycombs, The Journal of Strain Analysis for Engineering Design 35 (5) (2000) 383-388, doi: \bibinfo $\{$ doi $\}\{10.1243 / 0309324001514152\}$.

[34] L. J. Gibson, M. F. Ashby, The Mechanics of Three-Dimensional Cellular Materials, Proceedings of the Royal Society of London. A. Mathematical and Physical Sciences 382 (1782) (1982) 43-59, doi: \bibinfo $\{$ doi $\}\{10.1098 /$ rspa.1982.0088\}. 


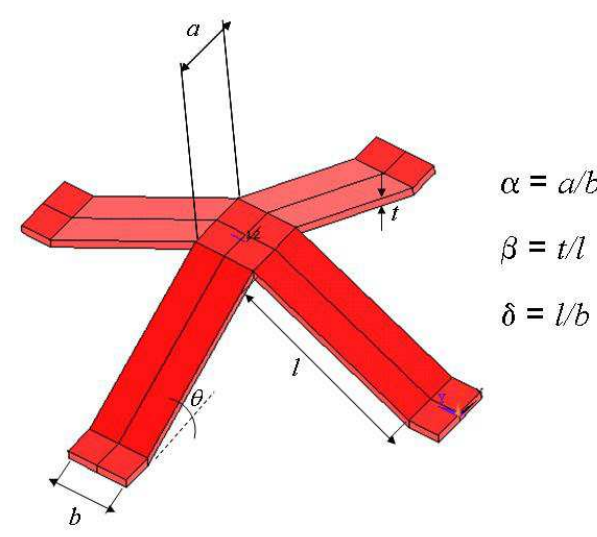

(a)

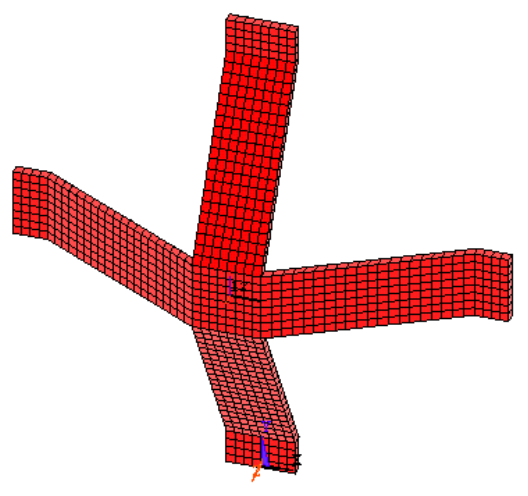

(b)

Figure 3: (a) RVE pf the pyramidal Kirigami core with its nondimensional geometry parameters; (c) FE RVE with $\alpha=1, \beta=0.05, \delta=6, \theta=20^{\circ}$

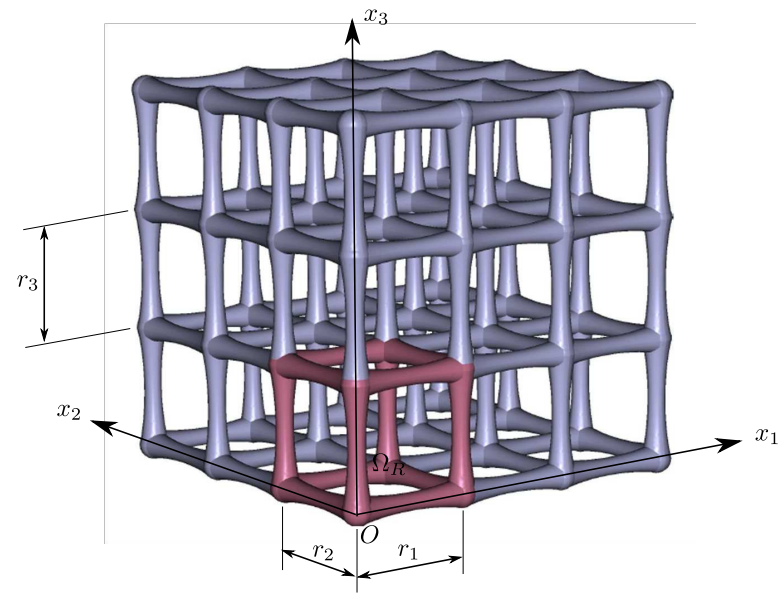

Figure 4: Generic 3D periodic cells 


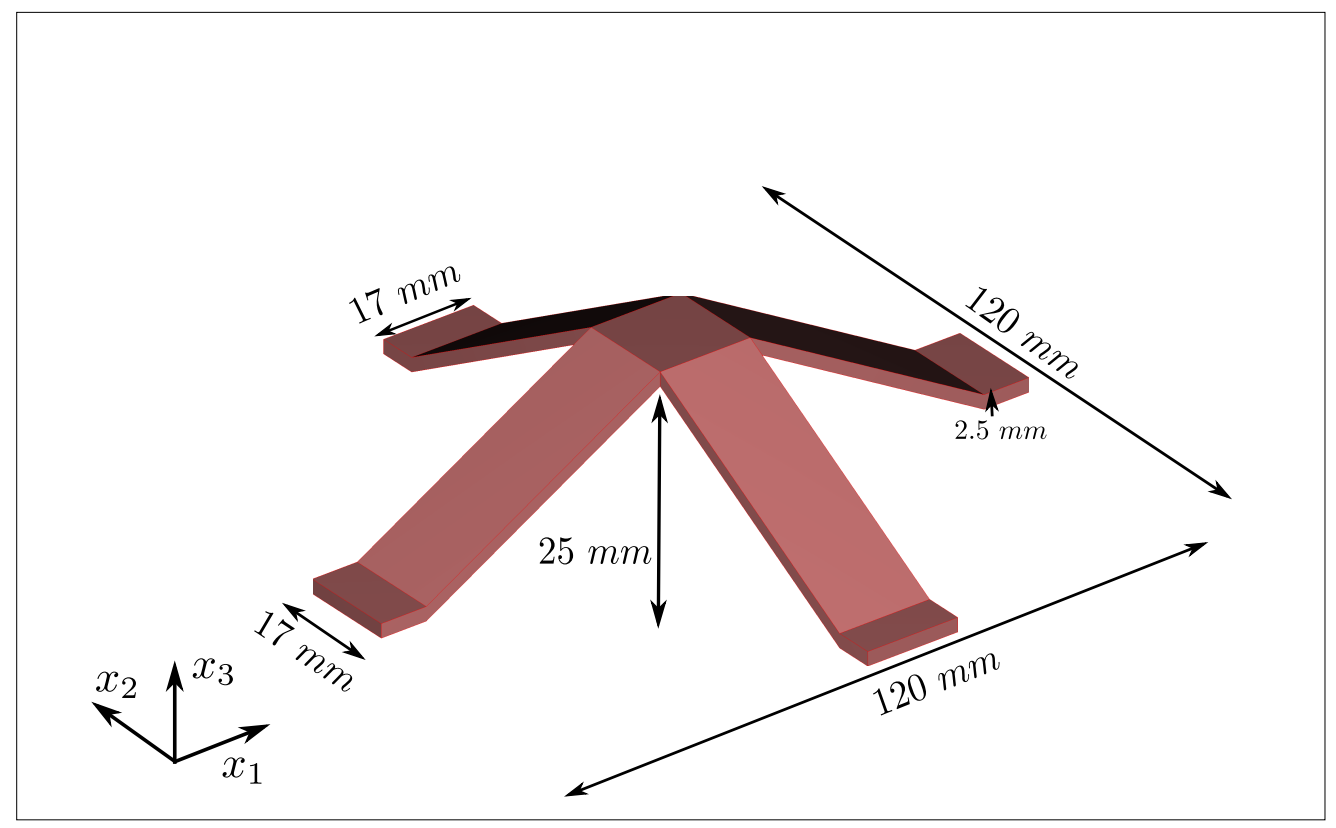

Figure 5: Layout of the damped Kirigami lattice unit cell considered for the 2D wave propagation study.

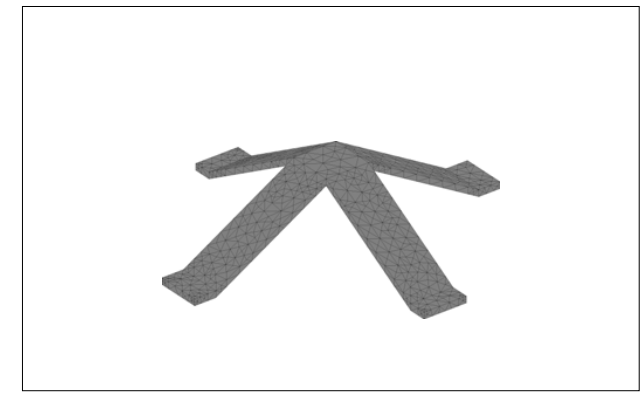

(a)

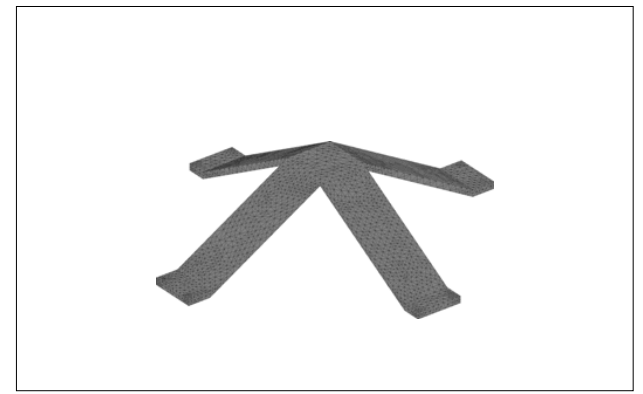

(b)

Figure 6: Unrefined (a) and refined (b) mesh cases 


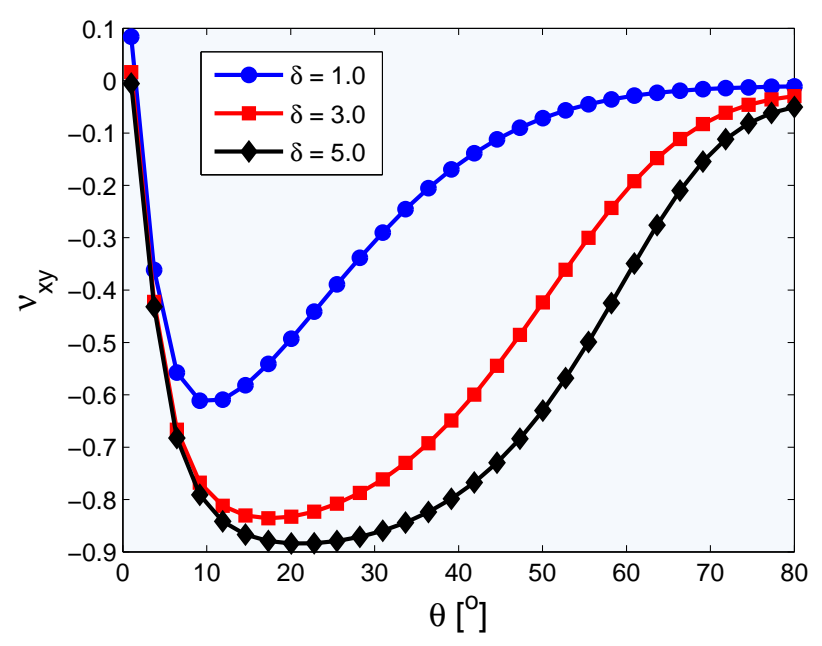

(a)

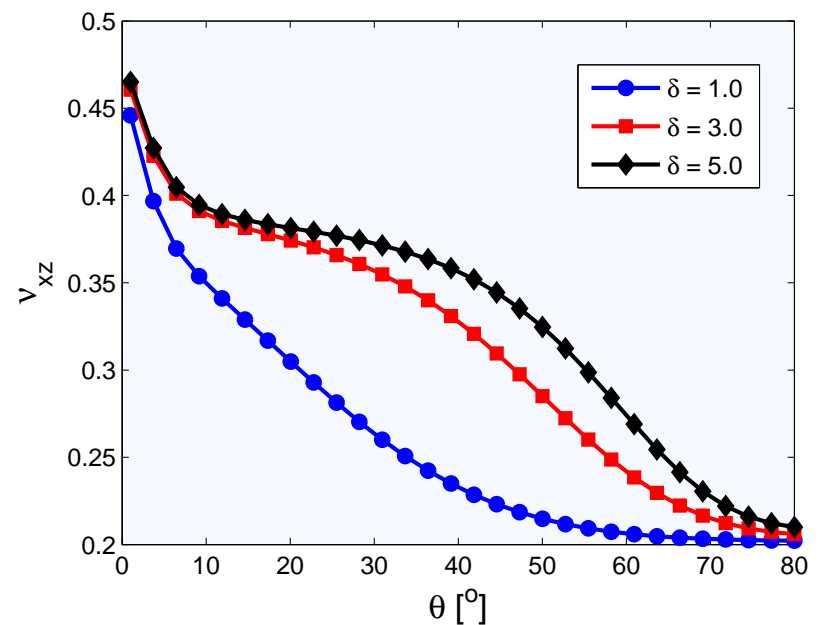

(b)

Figure 7: Distribution of the Poisson's ratios (a) $\nu_{x y}$ and (b) $\nu_{x z}$ versus the angle $\theta$ for different $\delta$ parameters, and $\alpha=1$. 


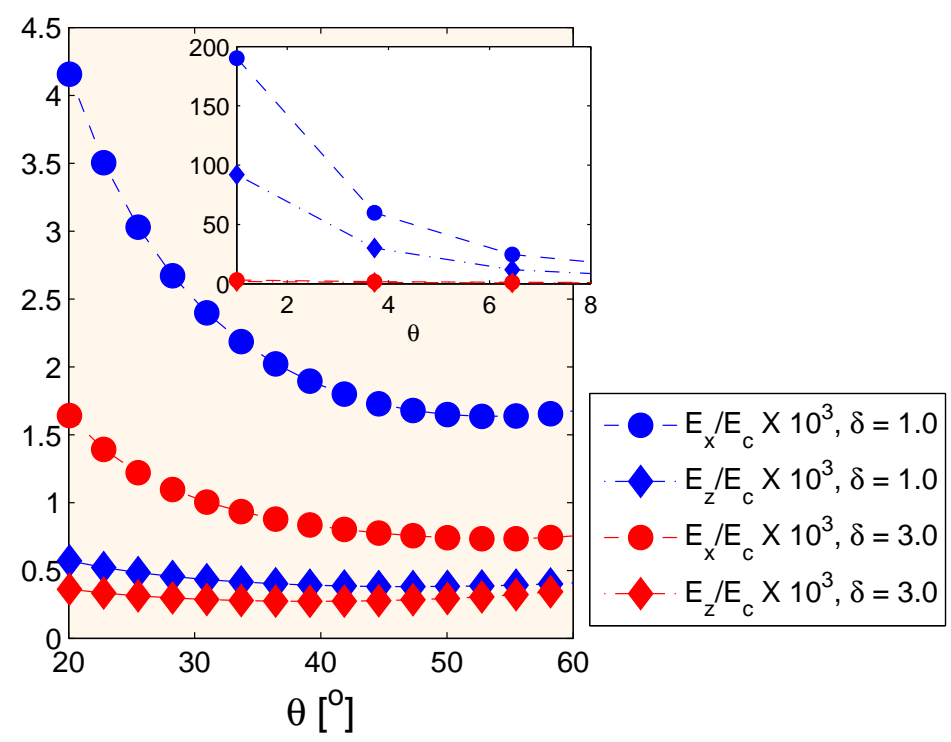

(a)

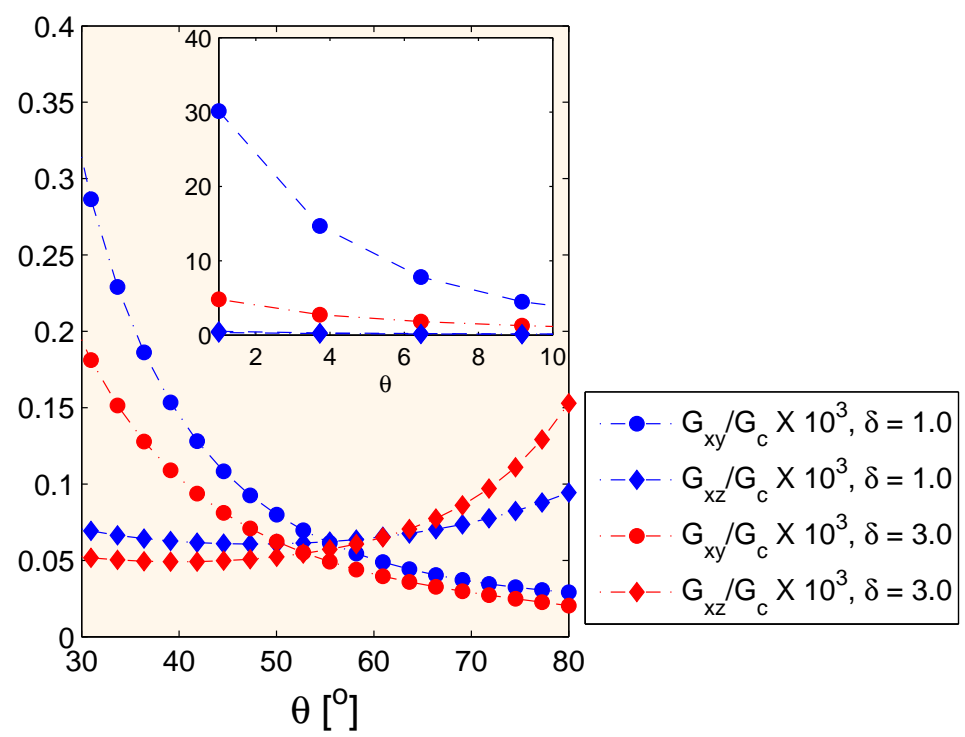

(b)

Figure 8: (a) Nondimensional in-plane and transverse Young's modulus versus the internal angle $\theta$ for different aspect ratios $\delta$; (b) Similar parametric curves for nondimensional in-plane shear and transverse modulus. For all calculations $\alpha=1$. 


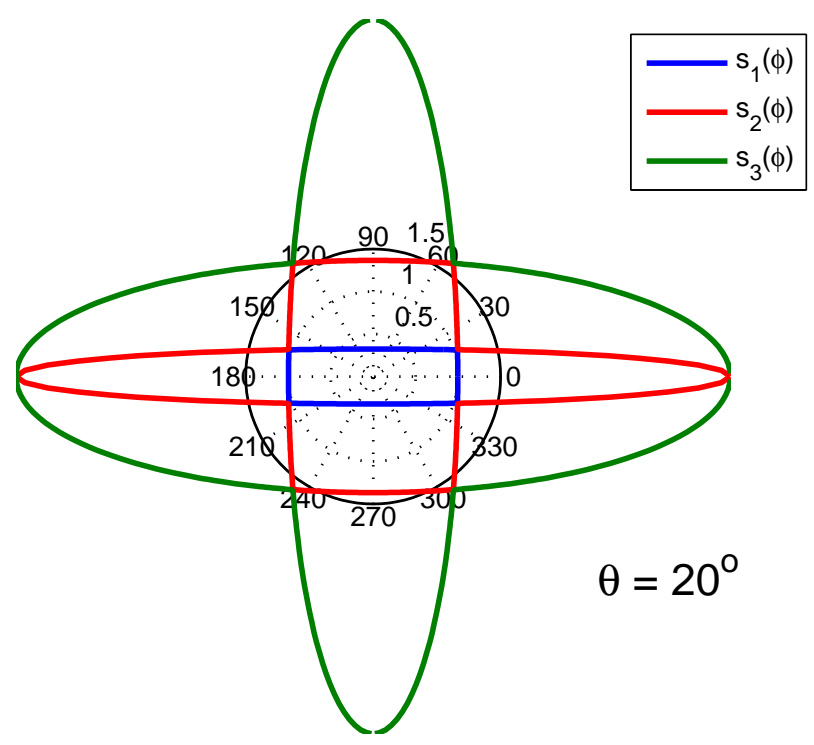

(a) $\delta=1.0$

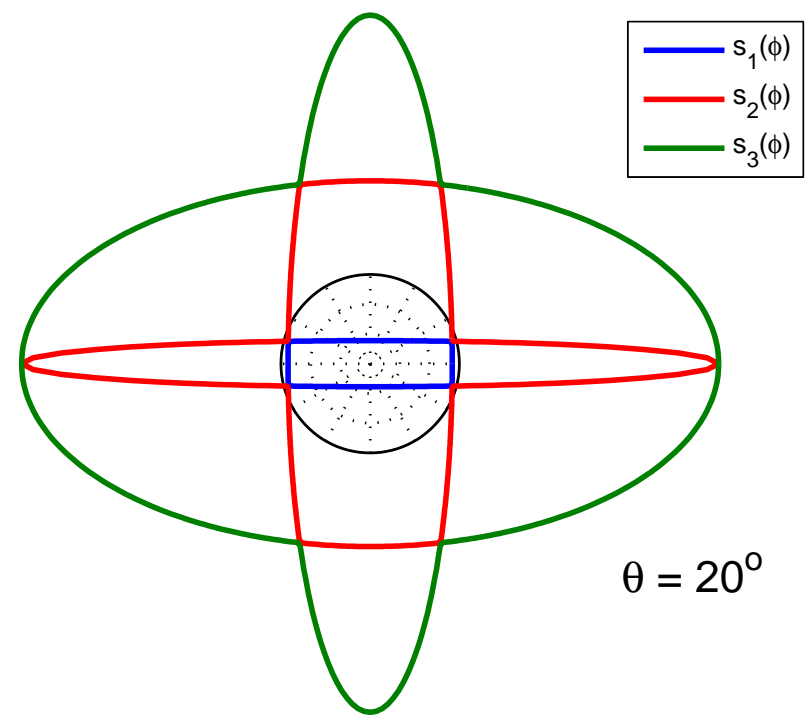

(b) $\delta=5.0$

Figure 9: Slowness curve in the $x z$ plane for $\alpha=1, \theta=20^{\circ}$ 


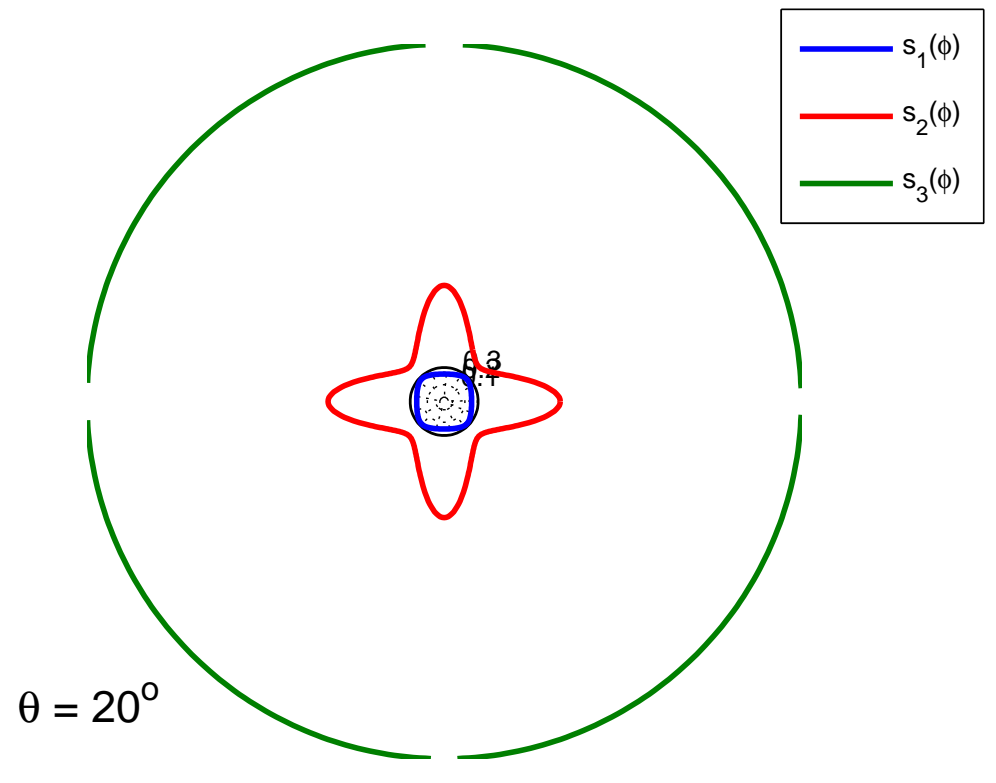

(a) $\delta=1.0$

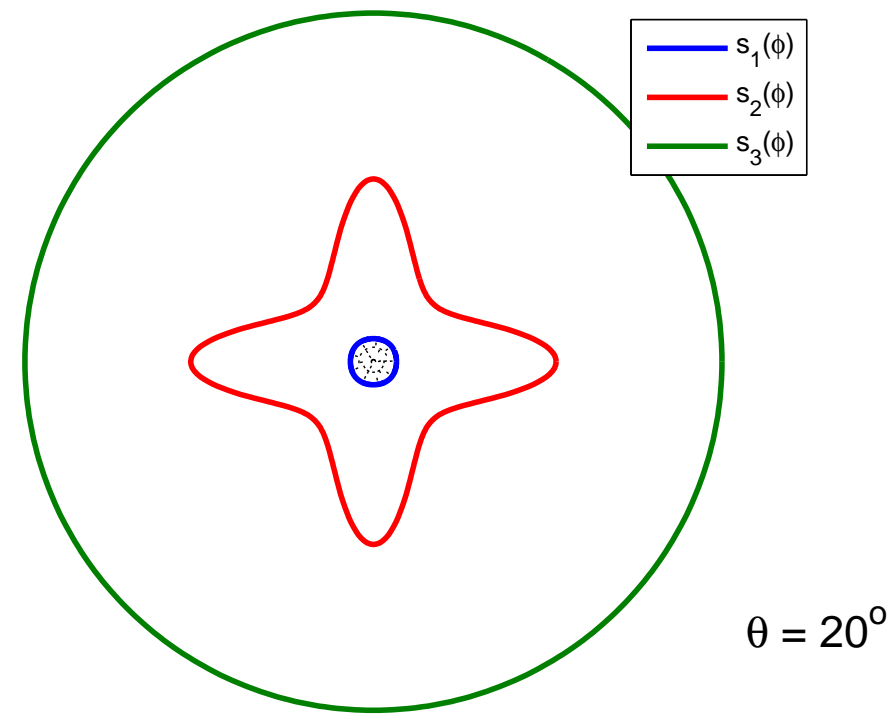

(b) $\delta=5.0$

Figure 10: Slowness curves in the $x y$ plane for $\alpha=1.0, \theta=20^{\circ}$ 


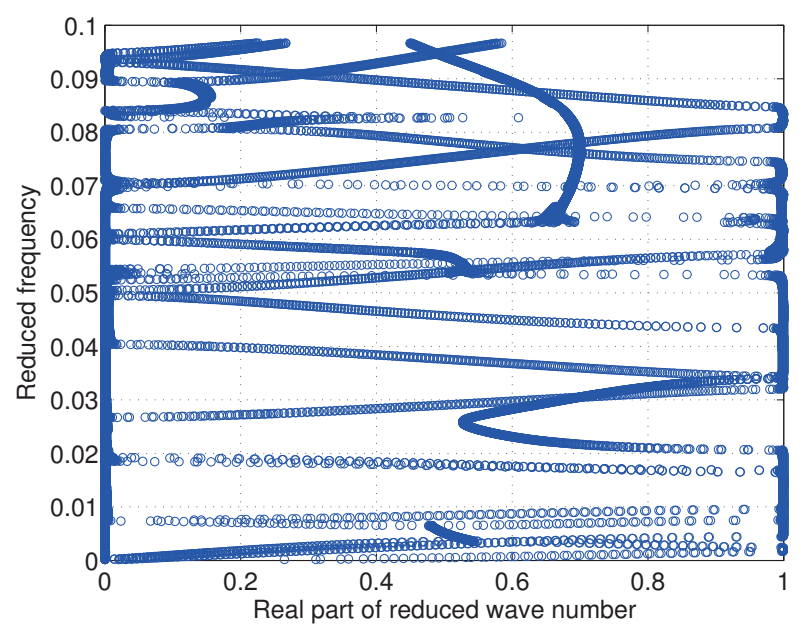

Figure 11: Dispersion curves of all modes of the studied system (imaginary part of $\lambda_{n}(\omega)$ or real part of $k_{n}(\omega)$.

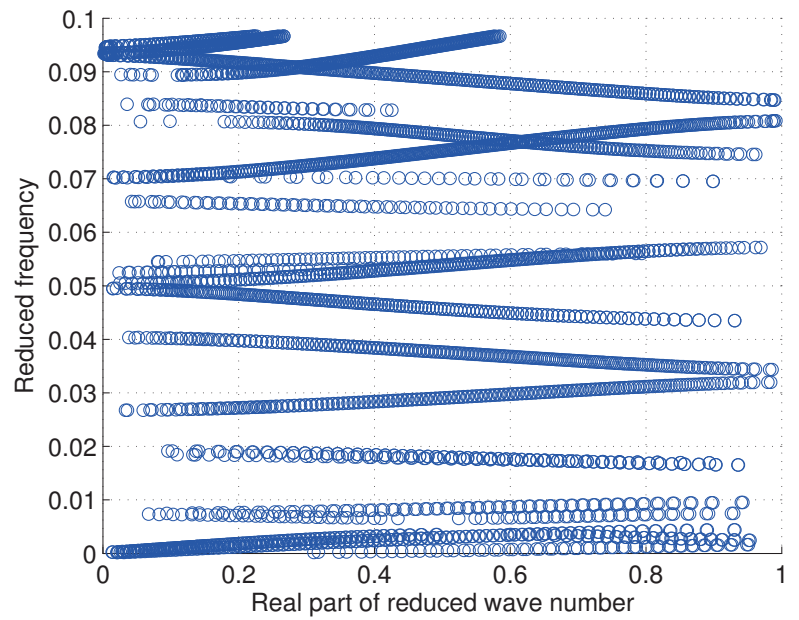

Figure 12: Dispersion curves of propagative modes of the studied system (imaginary part of $\lambda_{n}(\omega)$ or real part of $\left.k_{n}(\omega)\right)$. 


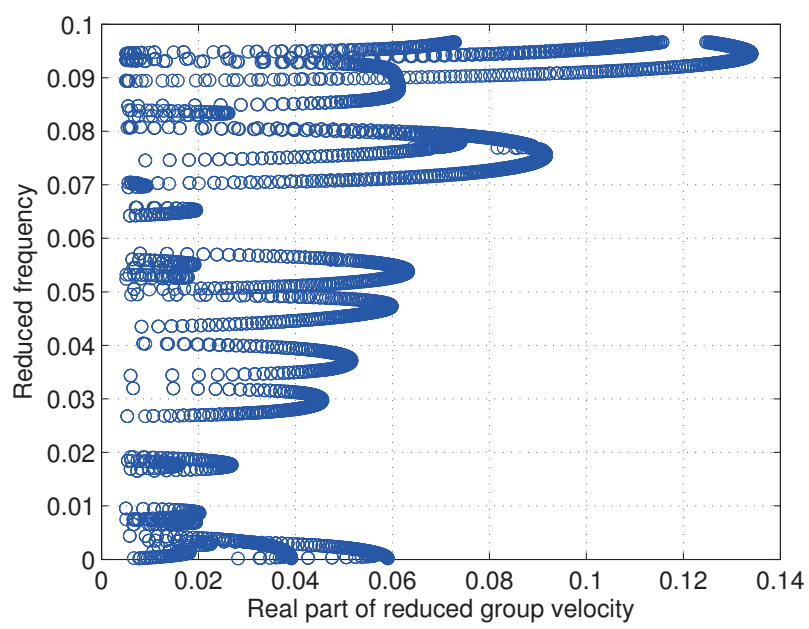

Figure 13: Dispersion curves of propagative modes of the studied system (imaginary part

$$
\text { of } \left.\lambda_{n}(\omega) \text { or real part of } k_{n}(\omega)\right) \text {. }
$$
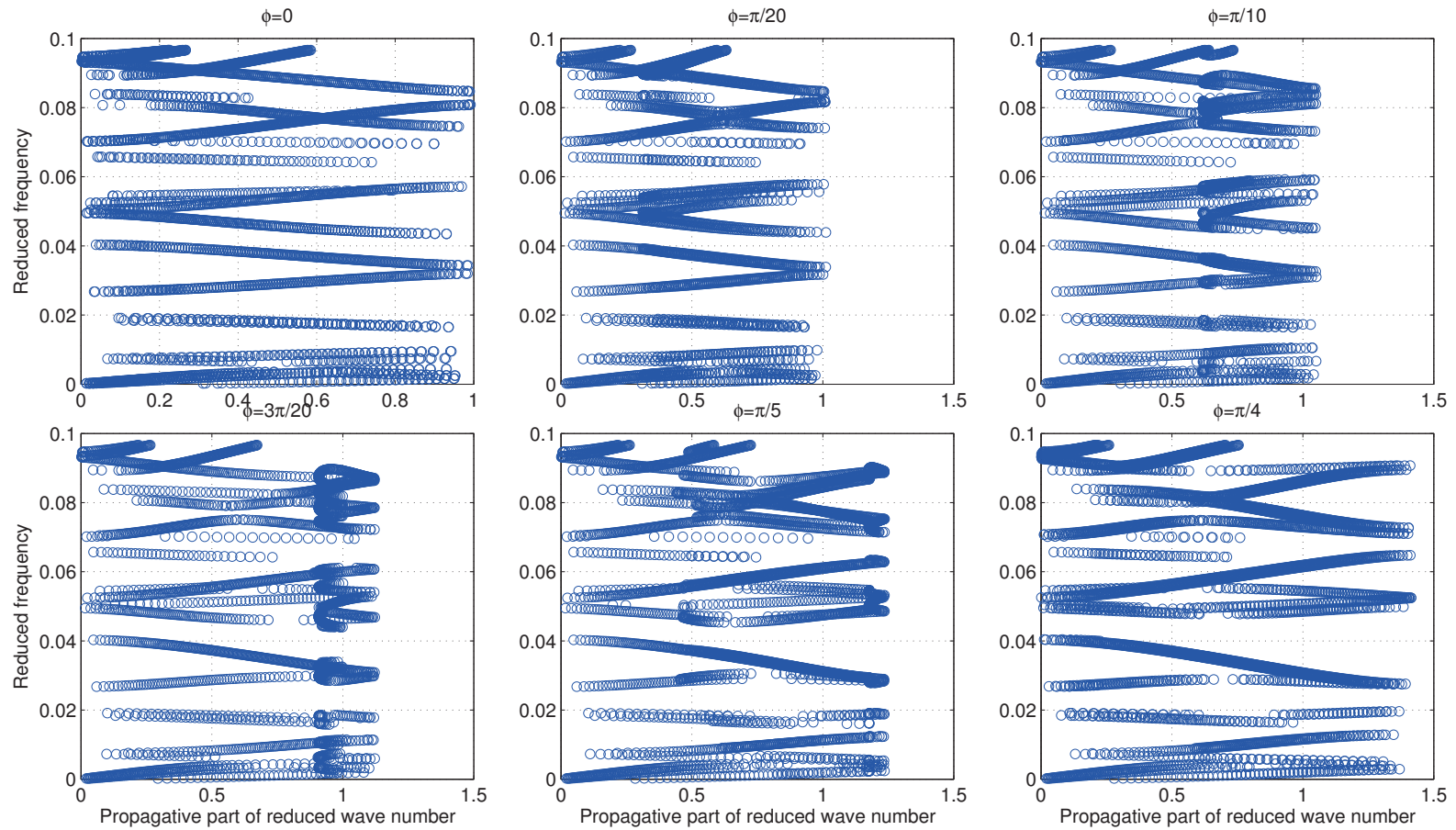

Figure 14: Propagative wave numbers of damped system $\left(\operatorname{Im}\left(\lambda_{n}(\omega)\right)\right.$ for different angles 


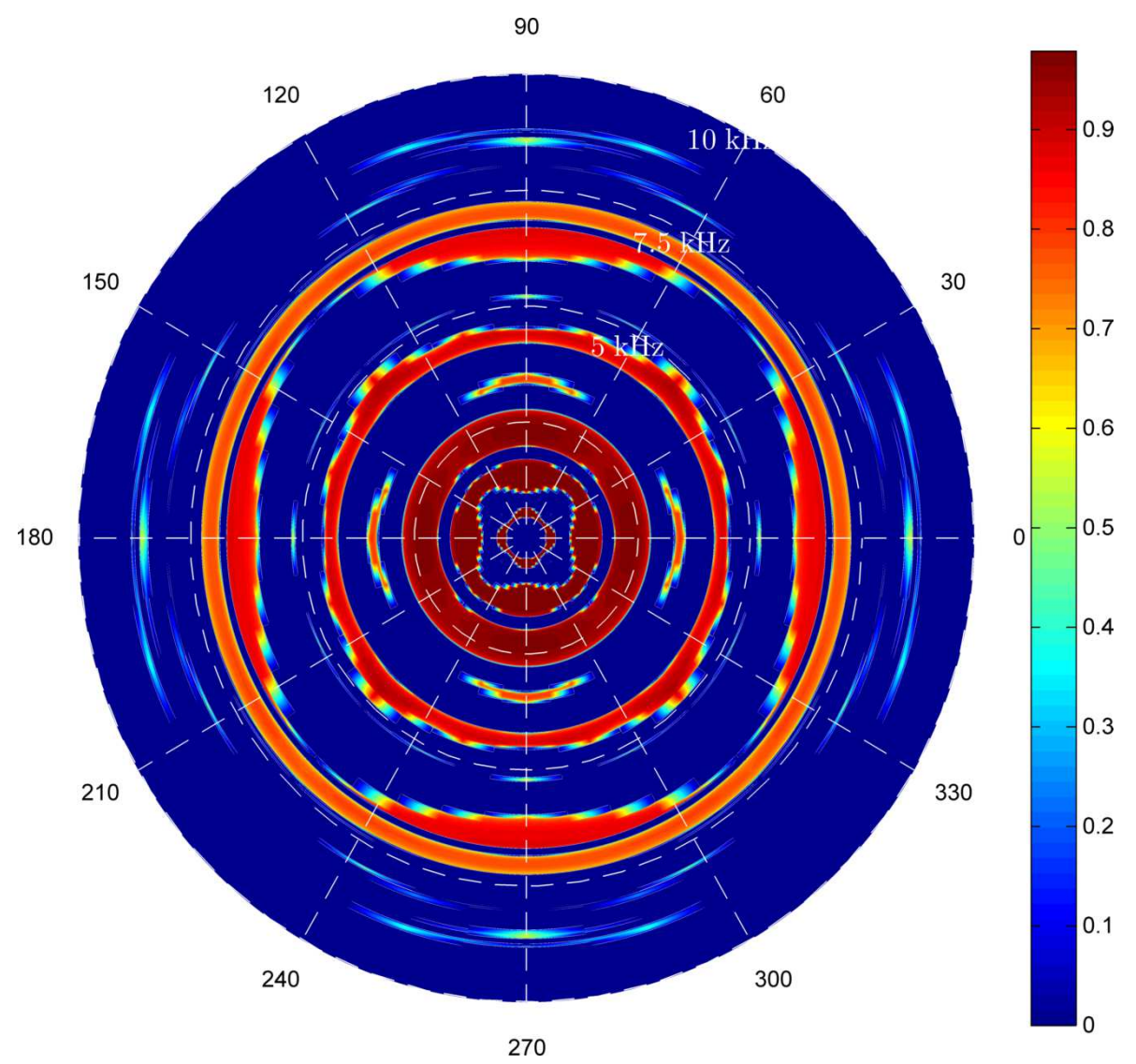

Figure 15: Directivity using the evanescence index (23) saturated at unit value 